\title{
Theory interpretations and challenges of DM searches
}

\section{Felix Kahlhoefer*}

Institute for Theoretical Particle Physics and Cosmology (TTK), RWTH Aachen University, D-52056 Aachen, Germany

E-mail: kahlhoefer@physik.rwth-aachen.de

The motivation for dark matter searches at the LHC and the models used to perform these searches are presented. A particular focus is placed on the advantages and disadvantages of renormalisable mediator models, followed by a discussion of their central predictions. The case of mono-Higgs signals is considered in further detail for illustration, followed by an outlook on the activities of the LHC Dark Matter Working Group.

Sixth Annual Conference on Large Hadron Collider Physics (LHCP2018)

4-9 June 2018

Bologna, Italy

${ }^{*}$ Speaker. 


\section{Introduction}

It is one of the great successes of modern physics that the cosmology of the Early Universe can be understood in terms of the properties of elementary particles. Even neutrinos, the most weaklyinteracting known particles, have an important impact on cosmological observables through their number, mass and interaction strength. These connections raise the hope that cosmology may also give us hints about yet undiscovered particles beyond the Standard Model (SM).

Indeed, an essential ingredient of Early Universe cosmology is dark matter (DM), which is assumed to create the potential wells in which stars and galaxies can form and thereby acts as the seed for structure formation. This very simple hypothesis produces a wealth of successful predictions, concerning for example the amount and distribution of structure in the present Universe. Most intriguingly, if interpreted in terms of particle physics, the DM hypothesis points towards the existence of a new stable particle.

At first sight, there is no obvious connection between these considerations and physics at the Large Hadron Collider (LHC), and indeed there are many models of DM that are fundamentally unobservable at the LHC [1]. Nevertheless, the LHC has a unique chance of discovering many of the theoretically most attractive DM models, which connect the DM problem to other unsolved issues of particle physics such as the hierarchy problem. These models of DM also tend to be the most predictive ones, because one can calculate the amount of DM produced in the early Universe via the freeze-out mechanism, which describes the decoupling of DM from thermal equilibrium.

The amount of DM in the present Universe is measured with remarkable precision, which imposes strong constraints on the parameter space of DM models. Indeed, DM particles produced via the freeze-out mechanism can neither be extremely heavy nor extremely weakly coupled and the most natural way to reproduce observations is to assume that DM particles have weak interactions and weak-scale mass (so-called WIMPs). In fact, WIMPs have exactly the right properties to be searched for at the LHC.

In order to devise and interpret DM searches at the LHC, we have to make assumptions on how DM particles interact with the SM. The simplest assumption would be that DM particles couple directly to SM particles (e.g. gauge bosons or Higgs bosons). This set-up, which is realised for example in certain Higgs portal models, faces however strong constraints from experiments (see e.g. $[2,3])$. To respect these constraints, it is commonly assumed that DM is uncharged under the SM gauge group and that there are no direct interactions between DM and SM particles. This leads to two possible avenues for constructing models of the interactions of DM.

The first direction is to consider non-renormalisable interactions, i.e. to parametrise the interactions of DM in terms of an unknown scale of new physics $\Lambda$. What makes this so-called effective field theory (EFT) approach so attractive is the finite number of possible operators and the very simple parameter space, which makes it possible to connect the constraints from different types of experiments. In practice, however, this approach breaks down for small $\Lambda$, in which case the EFT becomes invalid and does not capture all relevant features of the interactions.

These shortcomings can be addressed in an alternative approach by introducing additional particles that interact with SM particles and DM (so-called mediators). At first sight, this move comes at a high price, namely a substantial loss of generality due to the much larger number of possible directions, as well as an increased complexity of the parameter space for each model. 
Nevertheless, renormalisable mediator models possess a number of important advantages relative to the EFT approach:

- Renormalisable mediator models remain valid in all kinematic regions, such that it is straightforward to generate signal predictions and to include NLO corrections.

- Renormalisable mediator models potentially offer a more fundamental understanding of the underlying theory in the sense that one can compare different models in terms of their plausibility and complexity.

- Most importantly, renormalisable mediator models are at the same time flexible enough to predict a broad range of new signatures and predictive enough to allow to correlate different search channels in terms of the underlying structure of the theory.

In this presentation I will argue that it is precisely this third advantage that makes renormalisable mediator models such a compelling guide for DM searches at the LHC.

I will first review various DM models with new mediators and argue why it is important to consider renormalisable rather than simplified models. I will then detail the central predictions of renormalisable models based on a few concrete examples. Finally, I will provide an outlook on the future of LHC DM searches.

\section{DM models with new mediators}

To introduce interactions between the DM particle $\chi$ and SM particles, we can extend the SM by a mediator, which can have spin 1 :

$$
\begin{gathered}
\mathscr{L}_{\text {vector }}=-g_{\mathrm{DM}} Z_{\mu}^{\prime} \bar{\chi} \gamma^{\mu} \chi-g_{q} \sum_{q} Z_{\mu}^{\prime} \bar{q} \gamma^{\mu} q, \\
\mathscr{L}_{\text {axial-vector }}=-g_{\mathrm{DM}} Z_{\mu}^{\prime} \bar{\chi} \gamma^{\mu} \gamma_{5} \chi-g_{q} \sum_{q} Z_{\mu}^{\prime} \bar{q} \gamma^{\mu} \gamma_{5} q
\end{gathered}
$$

or spin 0 :

$$
\begin{gathered}
\mathscr{L}_{\text {scalar }}=-g_{\mathrm{DM}} \phi \bar{\chi} \chi-g_{q} \frac{\phi}{\sqrt{2}} \sum_{q} y_{q} \bar{q} q, \\
\mathscr{L}_{\text {pseudo-scalar }}=-i g_{\mathrm{DM}} \phi \bar{\chi} \gamma_{5} \chi-i g_{q} \frac{\phi}{\sqrt{2}} \sum_{q} y_{q} \bar{q} \gamma_{5} q .
\end{gathered}
$$

At first sight, all of these so-called simplified models appear renormalisable (since all couplings are dimensionless), but on closer inspection one discovers a lot of hidden complications.

For example, introducing a massive spin-1 mediator generally yields a non-renormalisable theory due to the longitudinal polarisation modes, which (at least for a mediator with axial couplings) do not decouple and violate unitarity at high energies [4]. Of course it is well-known how to make sense of such a theory by generating the mediator mass via the vacuum expectation value of an additional Higgs field. However, in such a set-up the additional Higgs boson is generally expected to couple to both the DM particle and to SM states, so that a consistent theory actually requires two mediators rather than one, each of which can give rise to new signatures [5]. 
The situation becomes even more complicated for the case of spin-0 mediators, which cannot directly couple to SM fermions in a gauge-invariant way. In the case of a scalar mediator, couplings can be introduced by Higgs mixing, at the cost of modifying the branching ratios of the SM Higgs boson and of introducing couplings between the mediator and SM gauge bosons. For a pseudoscalar such a mixing would violate $\mathrm{CP}$, such that it typically becomes necessary to introduce a second Higgs doublet. This additional Higgs doublet contains a pseudoscalar degree of freedom that can mix with the pseudoscalar mediator. Indeed, this realisation turns out to be the simplest one that does not suffer from unitarity violation in e.g. single-top production [6].

The discussion above clearly demonstrates that simplified DM models do not fully resolve the inadequacies of the EFT approach in the sense that unitarity violation may still be a problem at high energies. To address these issues one must introduce additional particles and structures in order to obtain models that are renormalisable and gauge-invariant. Imposing these requirements is of course not a necessity. Just like the EFT approach is appropriate to describe certain DM models in certain regions or parameter space, simplified models may correctly capture the phenomenology in the most important search channels. However, as will become clear in the following, these models miss a wealth of additional features, which arise naturally in renormalisable mediator models and are often irreducible. In other words, the predictions of renormalisable models allow to extend the DM search programme at the LHC and enhance experimental sensitivity.

\section{Central predictions of renormalisable models}

Renormalisable mediator models make two types of predictions: concerning the properties of SM particles and concerning processes involving new particles beyond the SM. This is different to both the EFT approach and the simplified model approach, which ignore modifications of the properties of SM particles and only contain predictions of the latter type. In consistent renormalisable models, on the other hand, these two types of predictions are typically inseparable.

For example, as mentioned above, a scalar mediator $s$ is expected to obtain couplings to SM particles via mixing with the SM Higgs boson. Such a mixing (parametrised by the mixing angle $\theta$ ) not only reduces the Higgs signal strength by a factor $\cos ^{2} \theta$, but may also induce an invisible decay mode of the SM-like Higgs boson proportional to $\sin ^{2} \theta$, which can be searches for in a range of different channels [7]. At the same time, if $s$ is sufficiently light, the decay $h \rightarrow s s$ followed by decays of $s$ into SM final states may lead to new exotic decay modes, such as $h \rightarrow 4 \ell$. Similar effects also occur in models with new spin-1 mediators and kinetic mixing.

But also predictions of the second type are very different in the case of renormalisable models, due to the richer internal structure of these models. Indeed, both EFTs and simplified models generically predict DM signals at the LHC to arise from the initial state radiation of SM particles (so-called mono-X signatures). Since the probability for QCD radiation is largest, one then finds that mono-jet processes are the most promising DM search channel, followed by mono-photon, mono-Z/W and mono-Higgs (in order of descending importance). This picture changes fundamentally in the case of renormalisable models, where SM particles can also be produced together with DM particles, either in the decays of heavier states or via final-state radiation. A few examples of such processes are shown in Fig. 1. 

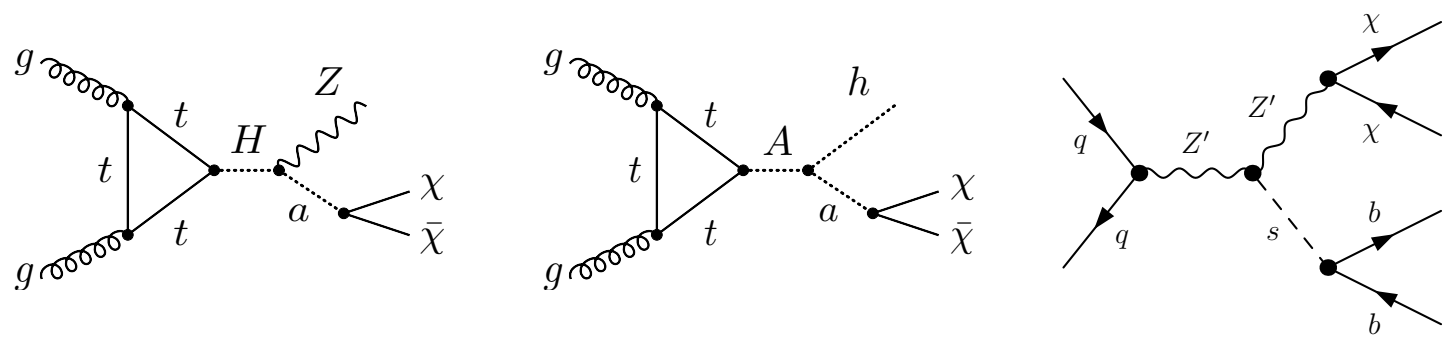

Figure 1: A few examples for processes that contribute to the production of DM at the LHC in the context of renormalisable mediator models.

The DM signatures arising from the diagrams in Fig. 1 differ fundamentally from mono-X signatures. First of all, the argument about the relative importance of different search channels no longer applies. The two diagrams on the left of Fig. 1, for example, lead to a substantial enhancement of the cross section for $Z+\mathbb{E}_{T}$ and $h+\mathbb{E}_{T}$, respectively. Moreover, kinematic distributions can be very different from the case of initial-state radiation, where one expects a monotonically falling $\mathbb{E}_{T}$ spectrum. For example, if the intermediate particle can be on-shell, one expects a Jacobian peak and correspondingly large amounts of missing transverse energy.

To illustrate these features, let us take a closer look at the mono-Higgs channel. In simplified models this channel is typically unimportant, as the probability for the emission of a Higgs boson from the initial state is tiny. Even for DM models with enhanced couplings to top quarks, the mono-Higgs channel is less important than for example $t \bar{t}+\mathscr{E}_{T}$. The situation can however change dramatically if Higgs bosons can be produced in the decays of a heavier particle. This is the case for example in DM extensions of Two Higgs Double Models, where the heavy pseudoscalar $A$ can decay into a SM Higgs boson and a pair of DM particles: $A \rightarrow h \chi \bar{\chi}$ (see central panel of Fig. 1). Indeed, it is perfectly conceivable that this is the dominant decay mode of $A$ and that traditional search channels like $t \bar{t}$ would be suppressed [8].

As can be seen in Fig. 2, searches for Higgs bosons in association with missing energy may hence give some of the strongest constraints on the parameter space of such models. Moreover, at least for the case that the Higgs boson is identified in the di-photon channel, the sensitivity of the LHC is still very much limited by statistics, such that substantial improvements can be expected for future searches [8].

\section{Outlook}

As the discussion above has indicated, extensions of Two Higgs Doublet Models with a pseudoscalar mediator and a DM particle have a very rich phenomenology with many different LHC signatures. Moreover, additional constraints on the model from electroweak precision measurements and flavour physics lead to an interesting interplay between different branches of particle physics. The price to pay is the considerable complexity of these models, which make it challenging to comprehensively explore the different allowed parameter regions and the corresponding kinematic distributions and search channels. The LHC Dark Matter Working Group is presently preparing a whitepaper with a set of recommended benchmark models [9]. This whitepaper will 

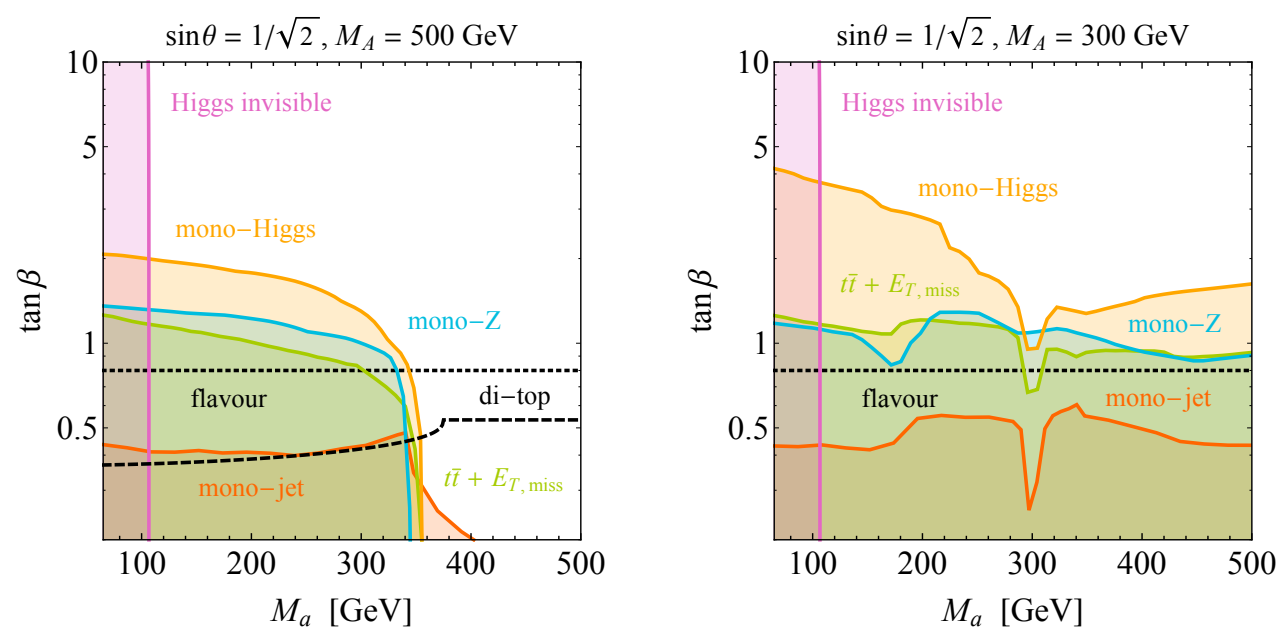

Figure 2: Constraints on the parameter space of an extension of a Two Higgs Doublet Model with a pseudoscalar mediator $a$ and a DM particle $\chi$. See Ref. [8] for details.

also provide an overview of ongoing efforts to study the DM phenomenology of these models, taking into account the relic density requirement as well as constraints from direct and indirect detection experiments.

The activities of the LHC DM working group reflect a broader shift of attention within the community from simple DM models to models of dark sectors, which typically require several new particles beyond the SM that cannot be arbitrarily heavy. These new particles can give rise to a wide range of exotic signatures, such as exotic resonances or displaced vertices in association with missing transverse energy (see e.g. Ref. [10]). Many of the potential signatures of dark sectors remain unexplored, so there remains much work to be done in order to fully exploit the potential of the LHC to discover DM.

\section{References}

[1] F. Kahlhoefer, On the LHC sensitivity for non-thermalised hidden sectors, Phys. Lett. B779 (2018) 388-392, [1801.07621].

[2] GAMBIT Collaboration, P. Athron et al., Status of the scalar singlet dark matter model, Eur. Phys. J. C77 (2017), no. 8 568, [1705.07931].

[3] P. Athron, J. M. Cornell, F. Kahlhoefer, J. Mckay, P. Scott, et al., Impact of vacuum stability, perturbativity and XENONIT on global fits of $\mathbb{Z}_{2}$ and $\mathbb{Z}_{3}$ scalar singlet dark matter, 1806.11281 .

[4] F. Kahlhoefer, K. Schmidt-Hoberg, T. Schwetz, and S. Vogl, Implications of unitarity and gauge invariance for simplified dark matter models, JHEP 02 (2016) 016, [1510 . 02110].

[5] M. Duerr, F. Kahlhoefer, K. Schmidt-Hoberg, T. Schwetz, and S. Vogl, How to save the WIMP: global analysis of a dark matter model with two s-channel mediators, JHEP 09 (2016) 042, [1606. 07609 ].

[6] P. Pani and G. Polesello, Dark matter production in association with a single top-quark at the LHC in a two-Higgs-doublet model with a pseudoscalar mediator, Phys. Dark Univ. 21 (2018) 8-15, [1712.03874]. 
[7] F. Kahlhoefer, Review of LHC Dark Matter Searches, Int. J. Mod. Phys. A32 (2017), no. 13 1730006, [1702.02430].

[8] M. Bauer, U. Haisch, and F. Kahlhoefer, Simplified dark matter models with two Higgs doublets: I. Pseudoscalar mediators, JHEP 05 (2017) 138, [1701 . 07427].

[9] LHC Dark Matter Working Group. In preparation.

[10] M. Duerr, A. Grohsjean, F. Kahlhoefer, B. Penning, K. Schmidt-Hoberg, et al., Hunting the dark Higgs, JHEP 04 (2017) 143, [1701.08780]. 